\title{
DNA damage as a marker of brain damage in individuals with history of concussions
}

\author{
Nicole Schwab ${ }^{1,2,3} \cdot$ Charles Tator $^{3,4} \cdot$ Lili-Naz Hazrati ${ }^{1,2,3}$
}

Received: 19 October 2018 / Revised: 27 November 2018 / Accepted: 28 November 2018 / Published online: 13 February 2019

(c) United States \& Canadian Academy of Pathology 2019

\begin{abstract}
Mild traumatic brain injury (mTBI) is common in many populations, including athletes, veterans, and domestic abuse victims. mTBI can cause chronic symptoms, including depression, irritability, memory problems, and attention deficits. A history of repetitive mTBI has been epidemiologically associated with developing early-onset dementia and neurodegenerative diseases and, in particular, is thought to be the underlying cause of chronic traumatic encephalopathy (CTE) - a progressive tauopathy diagnosed by the presence of perivascular hyperphosphorylated tau protein (p-tau) in the depths of cortical sulci. However, the scarce and focal pathology often seen in CTE does not correlate with the severity of symptoms experienced by patients. This paper proposes accumulation of $\gamma \mathrm{H} 2 \mathrm{AX}$, a marker of double-stranded DNA damage, as a novel pathological marker to identify brain damage post-mTBI. We present two cases of men with history of mTBI. Immunohistochemistry revealed extensive DNA damage throughout the frontal cortex, hippocampus, and brainstem areas. Furthermore, gene expression profiling showed increases of ataxia telangiectasia mutated (ATM) and checkpoint kinase 2 (CHEK2), two serine/threonine kinases recruited in response to double-strand breaks in the DNA damage response pathway. These cases highlight the complex pathophysiology of head trauma, and suggest DNA damage as the molecular mechanism behind mTBI-induced pathology and symptoms.
\end{abstract}

\section{Introduction}

Mild traumatic brain injury (mTBI) can have devastating effects long-term on brain health. However, the pathophysiological mechanism by which mild trauma induces brain dysfunction remains unknown. Several post-mortem case series have shown that recurrent mTBI can lead to a neurodegenerative disease called chronic traumatic encephalopathy (CTE) that is characterized by the accumulation of hyperphosphorylated tau (p-tau) in a specific pattern in the brain. However, the underlying molecular pathways leading

Lili-Naz Hazrati

lili-naz.hazrati@sickkids.ca

1 Department of Laboratory Medicine and Pathobiology, University of Toronto, Toronto, ON, Canada

2 The Hospital for Sick Children, Toronto, ON, Canada

3 Canadian Concussion Centre, Toronto Western Hospital, Toronto, ON, Canada

4 Division of Neurosurgery, University Health Network, Toronto Western Hospital, University of Toronto, Toronto, ON, Canada to this progressive neurodegenerative disease is very limited, and there are likely other pathological hallmarks which may be better markers of brain damage in mTBI and provide stronger indications on the pathophysiology of the disease. This paper will summarize the known neuropathology of CTE and will detail two cases of individuals with a history of mTBI in order to introduce a novel marker of cellular damage in athletes with history of mTBI.

\section{Mild traumatic brain injury}

Mild traumatic brain injury (mTBI), which includes concussions and subconcussive hits, is defined by the World Health Organization as an acute brain injury caused by mechanical force to the head [1]. It has a high incidence and prevalence throughout the world and, in particular, occurs frequently and repetitively in subpopulations such as contact sports players (including American football, hockey, rugby, and boxing), survivors of domestic abuse, and military veterans [2]. Often mTBIs go unnoticed or unreported, making the true incidence of mTBI likely much higher than reported in literature [3]. Studies in postconcussive clinical presentation utilize self-report measures 
[4], and lack consensus due to the fact that mTBI outcomes vary significantly between individuals [5]. Despite this variation, chronic exposure to mTBI can be linked to persisting symptoms in some individuals including depression, anxiety, short-term memory problems, suicidality, sleep disturbance, attention deficits among others and ultimately dementia $[6,7]$.

Epidemiological studies have identified mTBI as a primary risk factor for early-onset dementia and neurodegenerative diseases including Alzheimer's disease (AD) [8], Parkinson's disease (PD) [9], frontotemporal dementia (FTD) [10], amyotrophic lateral sclerosis (ALS) [11], and Diffuse Lewy body disease (DLBD) [12]. A 2017 nationwide cohort study of over 300,000 individuals in Sweden by Nordstrom and Nordstrom [13] found a significant association between multiple TBIs and dementia diagnosis, and an increased risk of developing dementia in pairs of siblings with discordant TBI history. In the US, a study of over 350,000 veterans with history of TBI found that mTBI without loss of consciousness was associated with more than a two-fold increase in risk of being diagnosed with dementia later in life [14]. They reported the risk to develop dementia jumping from $2.6 \%$ without mTBI to $6.1 \%$ with mTBI.

\section{Neuropathology of CTE}

In 2013, McKee et al. produced a large case series of 85 individuals with a history of mTBI and found a spectrum of distribution of $\mathrm{p}$-tau ranging from perivascular lesions to neurofibrillary tangles (NFTs) throughout the cortex and medial temporal lobes, TAR DNA-binding protein 43 (TDP-43) inclusions and neurites, and a spectrum of clinical symptoms ranging in severity [15]. From this study, McKee and colleagues suggested that CTE is a progressive neurodegenerative disease, for which the degree of pathological burden corresponded with symptom severity [15]. However, they did also attribute symptomatology in cases where the pathology was very scarce and focal. More recently in $2017 \mathrm{Mez}$ et al., also from Boston University, published a case series of 202 football players of various playing levels, the largest case series on CTE to date [16]. This study diagnosed CTE by the presence of perivascular p-tau lesions in the depths of cortical sulci and found that 177 (87\%) subjects presented with CTE.

In 2016, McKee and colleagues published the first ever consensus criteria, with UNITE and the NINDS/NIBIB panel, for the neuropathological diagnosis of CTE [17]. These criteria were produced by researchers at Boston University along with seven neuropathologists experienced in neurodegenerative diseases. In this meeting, the pathologists blindly evaluated 25 cases of various tauopathies, including 10 cases of CTE. The panel reached preliminary consensus regarding the pathology of these cases and, in particular, agreed on the diagnoses of the 10 CTE cases.

\section{Gross Pathology of CTE}

In early CTE, there is generally no evidence of trauma to the brain and the brain appears normal macroscopically [15]. In more advanced CTE, macroscopic findings may include ventricular dilation, a cavum septum pellucidum, septum fenestration, medial temporal lobe atrophy, and pallor of the substantia nigra and locus coeruleus [15]. The diagnosis of CTE, however, is based only on microscopic/ immunohistochemical features of p-tau distribution [17].

\section{Microscopic Pathology of CTE}

The 2016 consensus group identified one feature required for the diagnosis of CTE: the presence of p-tau aggregates in neurons and astrocytes, specifically in perivascular regions at the depths of cortical sulci [17]. One focus of perivascular tau in the depth of a cortical sulcus is sufficient for post-mortem diagnosis of CTE, as there is no lower bound to this criteria. In contrast to other tauopathies, such as $\mathrm{AD}$, where higher stages of the disease are correlated with clinical symptoms, in CTE any amount of pathology is proposed to be the underlying driver of neurobehavioral changes. In CTE tau aggregates are found in neurons and glial cells rather than solely neurons [15]. The pathology starts in the depths of sulci in the frontal lobe rather than in the medial temporal lobes as is the case in AD [18]. It has been suggested that as the disease progresses, p-tau aggregates spread throughout the cortex and to the medial temporal lobes [15].

This consensus meeting also identified five tau-related supportive neuropathological features of CTE diagnosis, and two non-tau-related supportive features. The tau-related features included the following: (1) p-tau pretangles and/or NFTs in the cortical superficial layers II-III, (2) p-tau pretangles and/or NFTs preferentially affecting CA2 and dendritic swellings in CA4 of the hippocampus, (3) immunoreactivity in neurons and astrocytes of subcortical nuclei including the mammillary bodies, amygdala, nucleus accumbens, thalamus, midbrain tegmentum, nucleus basalis of Meynert, raphe nuclei, substantia nigra, and locus coeruleus, (4) immunoreactivity in astrocytes in the subpial and periventricular regions, and (5) immunoreactive grain-like and dot-like structures. The non-tau-related supportive diagnostic features are as follows: (1) gross microscopic features as described previously, and (2) TDP-43 neuronal inclusions and dot-like structures in the hippocampus, temporal cortex, and the amygdala. 


\section{Beta-amyloid after mTBI}

In Boston University's 2017 case series of 202 football players, $61 \%$ of subjects diagnosed with CTE presented with diffuse or dense core senile $A \beta$ plaques [16]. Further, a 2015 case series by Stein et al. [19] similarly reported that $52 \%$ of CTE subjects presented with A $\beta$ pathology, either as diffuse or senile plaques. This paper also found that when controlling for age, the presence of $\mathrm{A} \beta$ plaques was associated with worsened dementia, co-morbid LBD, and more severe CTE p-tau pathology, although for the latter it is unclear if they refer to an increase of p-tau burden or to the number of peri-vascular $\mathrm{p}$-tau foci. This raises the possibility that cases presenting with both $p$-tau and $A \beta$ represent co-existing $\mathrm{AD}$, and that $\mathrm{AD}$ is the true driver of symptoms. This possibility is supported by the fact that the chances of an individual in this cohort having dementia was 3.9 times higher in cases with diffuse $\mathrm{A} \beta$ plaques and 4.5 times higher in individuals with neuritic plaques compared to those without any $A \beta$ pathology when controlling for age. Individuals with $A \beta$ pathology also tended to present with Parkinsonism and motor symptoms more frequently than those without $A \beta$ plaques [19]. Although $A \beta$ plaques are a hallmark feature of $\mathrm{AD}$ [20] and this data may seem reflective of comorbidities, it is argued that amyloid precursor protein (APP) accumulates rapidly in injured axons after acute TBI and this might be a possible precursor to $\mathrm{A} \beta$ deposition [21]. However, axonal injury is not a consistent reported feature of mTBI.

\section{a-synuclein after mTBI}

$\alpha$-synuclein is a soluble protein involved in synaptic transmission and is found as insoluble aggregates in various synucleinopathies, including PD [22]. Accumulation of $\alpha-$ synuclein has been found after acute TBI in mice [23], and in the cerebro-spinal fluid (CSF) of patients after severe TBI [24]. In McKee et al,'s case series in 2013, $\alpha$-synuclein was found in $15(22 \%)$ of all CTE cases analyzed, primarily in the olfactory bulb and medulla [15]. In a case series of 269 contact sports players, Adams et al. found that the number of years an individual is exposed to head trauma in sports predicted the development of Lewy bodies (LB) in the neocortex [12]. The neocortical LB was also associated with increased $\mathrm{A} \beta$ plaques and $\mathrm{p}$-tau pathology, and the authors speculated that LB load accounted for many of the motor symptoms reported in CTE, although cortical LBs are typically strong drivers of dementia, as seen in DLBD [25].

\section{TDP-43 after mTBI}

TDP-43 is an RNA binding protein which becomes phosphorylated, ubiquitinated, and cleaved in a disease state, such as ALS or FTD [26]. In CTE, TDP-43 pathology is very common and is considered one of the supporting features of diagnosis [17]. In their 2013 case series, McKee et al. found TDP-43 positive cytoplasmic inclusions in 58/68 (85.3\%) of CTE cases [15]. Additionally, in the 2017 case series out of Boston University on American football players, 85 (48\%) cases of CTE presented with TDP-43 immunopositive neurites and inclusions, although they did not specify the distribution of this pathology [16].

\section{Proteinopathies are end-points and do not define underlying pathophysiology}

Despite the fact that the diagnostic criteria for CTE only requires perivascular $\mathrm{p}$-tau accumulation in the depths of cortical sulci, it is clear from the literature that the pathology of CTE is much more complex. Accumulation of misfolded proteins is quite common in neurodegenerative diseases [27], for example beta amyloid $(\mathrm{A} \beta)$ in $\mathrm{AD}$ [20], asynuclein in PD [28], and TDP-43 in FTD and ALS [29]. Interestingly, brains of individuals with history of mTBI display all three of these proteinopathies [23, 30, 31], making the neuropathology of head trauma broad and diverse in nature. It may therefore be helpful to look at CTE and mTBI-induced brain damage as a condition presenting with polypathology with co-existence of different proteinopathies [32]. It is possible that mTBI induces, in some people, a neurodegenerative process characterized by misfolded proteins but not limited to tauopathy. The perivascular tau pathology as the unique signature of mTBIinduced brain disease appears non-specific as this pattern is described in brains with no history of trauma [33] and could be confused with other non-mTBI related pathologies such aging-related tau astrogliopathy (ARTAG) [34]. Moreover, when the pathology is minimal and focal, it becomes very implausible to explain all the patient's symptoms. Epidemiological studies as stated above do link mTBI to a higher risk of developing a form of neurodegenerative disease later in life either as a classical disease such as $\mathrm{AD}$ or a more complex mixed disease with numerous abnormal protein aggregates such as p-tau, TDP-43, alpha-synuclein and A $\beta$. We propose that the abnormal proteinopathies reflect the endpoint of more complex underlying pathophysiological process that remains to be determined. Extensive focus on abnormal proteins is limiting the development of proper biomarkers and potential treatments in living individuals. Indeed, clinically, most of the symptoms associated with trauma induced brain pathology are not unique to the disease and could be reflective of other conditions [35], making diagnosis in a living person unattainable. Identification of the initiating molecular process(es) that may evolve to lead to neurodegenerative disease will open the 
door to more effective interventions in patients suffering from ill-effects of multiple concussions.

\section{DNA damage as a marker of mTBI-induced brain damage}

The p-tau pathology reported in early stages of CTE tends to be extremely focal and scarce [15] and is therefore unlikely to be the cause of widespread, severe symptoms reported by affected individuals [36]. It is possible, that there is another pathological phenomenon underlying the clinical presentation of individuals with mTBI-induced brain dysfunction which either precedes or is independent of p-tau pathology.

In general, DNA repair deficiency underlies many neurological diseases in both humans and mice [37]. Deficient DNA repair pathways have been implicated in neuronal dysfunction [38] and has been proposed as an important factor in premature aging and neurodegeneration [18]. For example, in a postmortem study on $11 \mathrm{AD}$ patients and eight controls, Mullaart and colleagues found two times more DNA damage in the cortex of individuals with $\mathrm{AD}$ than controls [39]. Similarly, AD brains have been shown to have decreased base-excision repair activity and accumulation of double-stranded DNA breaks [40]. In multiple neurodegenerative diseases including $\mathrm{AD}, \mathrm{PD}$, and ALS, not only is the accumulation of DNA damage found, but has been suggested as a contributing factor to the progression of disease [41].

Following double-stranded DNA breaks, H2A histone family member $\mathrm{X}(\mathrm{H} 2 \mathrm{AX})$ becomes phosphorylated at Ser139 to become $\gamma \mathrm{H} 2 \mathrm{AX}$ [42]. This event is one part of the DNA damage response (DDR), an endogenous molecular cascade which exists to repair the thousands of DNA lesions cells experience daily [43]. DNA damage can occur as a result of exogenous factors like UV radiation, toxic chemicals, and oxidative stress, and endogenously through replication errors and metabolic reactive oxygen species (ROS) production [18]. Although accumulation of DNA damage after mTBI has not yet been explored in humans, it has been shown that repeated blast exposures lead to widespread DNA damage in mice [44] and that accumulation of cell-free DNA in blood can be used as a predictor of neurological outcome after mTBI in rats [45]. Accumulation of DNA damage after mTBI may therefore be sufficient to explain the symptoms in individuals who are found to have relatively scarce proteinopathy.

$\gamma \mathrm{H} 2 \mathrm{AX}$ has been shown to be a sensitive and specific marker of DSBs both in vitro and in vivo [46], and its detection to mark DNA damage is considered superior to many other techniques, including comet assays and pulsefield gel electrophoresis [47]. Background levels of $\gamma \mathrm{H} 2 \mathrm{AX}$ have also been observed in populations of dividing cells [48], reflecting accumulation of DNA breaks during Sphase [49], although this is not the case in non-dividing cells such as neurons. Although $\mathrm{H} 2 \mathrm{AX}$ has more than one serine residue capable of phosphorylation, phosphorylation at Ser139 to form $\gamma \mathrm{H} 2 \mathrm{AX}$ is specific to DNA damage [46], and up to thousands of $\gamma \mathrm{H} 2 \mathrm{AX}$ proteins are formed for every DSB [50]. $\gamma \mathrm{H} 2 \mathrm{AX}$ is therefore a specific marker of DNA damage and does not represent other phosphorylation events. It is important to note that the appearance of $\gamma \mathrm{H} 2 \mathrm{AX}$ foci differs between techniques. For example, $\gamma \mathrm{H} 2 \mathrm{AX}$ foci tend to appear punctuated and dot-like when using immunofluorescence [51], whereas the stain appears fairly diffuse and uniform when performed with immunoperoxidase [52], as used here. A limitation of using immunohistochemistry is the inability to count individual DSBs, as is possible with immunofluorescence, however it is still considered a reliable marker of DNA damage [46] especially when combined with the detection of other markers of DNA damage. Phosphorylation of H2AX into $\gamma \mathrm{H} 2 \mathrm{AX}$ occurs through the actions of two serine/threonine kinases, ataxia telangiectasia mutated (ATM) and ATM-RAD3-related (ATR), both of which are activated following DNA damage and cellular stressors [53]. ATM is the main mediator of the DDR and is promptly activated via auto-phosphorylation following DNA damage, specifically in the form of DSBs, and activates downstream serine/threonine kinase chekpoint kinase 2 (CHEK2) to mediate DNA repair pathways [54, 55]. Upregulation of ATM, CHEK2, and simultaneous presence of $\gamma \mathrm{H} 2 \mathrm{AX}$ foci are strong, specific, and sensitive indicator of DNA damage in the form of DSBs.

Here, through two cases as examples, we propose that repetitive mTBI causes widespread DNA damage, which may be the underlying explanation of neurological symptoms, and that markers of DNA damage are better suited for evaluating brain damage after mTBI. DNA damage can become persistent, spread to more areas and lead to neurodegenerative disease later in life, most likely dependent on different individual capabilities to efficiently repair the DNA damage.

\section{Case examples}

The complexity of neuropathologically diagnosing CTE and establishing the cause of this pathology can be illustrated with the following post-mortem human cases. The brains of these individuals were received for neuropathological consultation and underwent full neuropathological assessment.

\section{Clinical history}

\section{Case 1}

This subject was a 33 year old man with a history of mTBI due to his long-term involvement with mixed martial arts. 
Fig. 1 Gross and microscopic features of case 1 . Panels a-c show macroscopic features of the brain with bilateral old orbito-frontal contusions. Panel c shows a coronal cut of the brain without any evident gross pathological changes. Panel d shows a whole slide scan of the hippocampus with "a" and "b" showing the location of p-tau positive profiles and further shown respectively in panels (e and f) at high power. Scale in (e) is $200 \mu \mathrm{m}$ and in (f) is $2 \mathrm{~mm}$

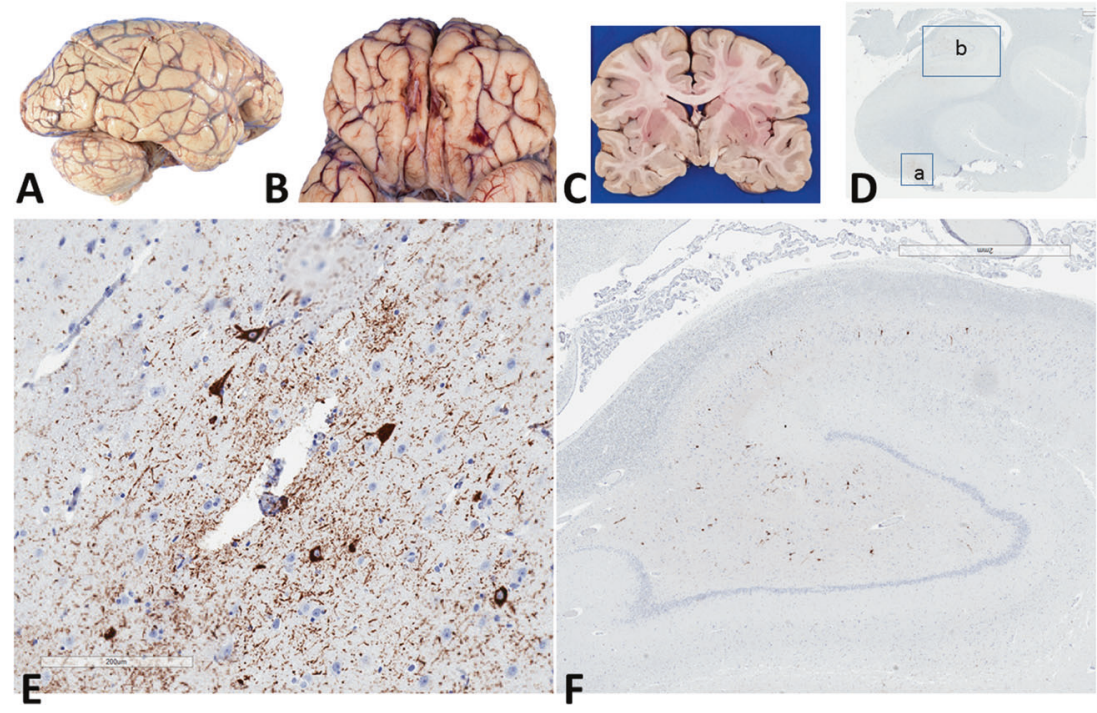

The individual was detained by police after a low-velocity car collision, and died suddenly due to cardiac arrest with acute concussion sustained during a brief altercation during his arrest. Resuscitation was unsuccessful and there was no survival or hospitalization. He also had a history of drug abuse (cocaine) and alcohol abuse. At autopsy he was diagnosed with cardiomegaly (abnormal enlargement of the heart) and cause of death attributed to a cardiac event.

\section{Case 2}

This subject was an 82 year old man with a long-term history of traumatic brain injury due to his long-term career in the Canadian Football League (CFL). He had a history of hypertension, hyperlipidemia, and diabetes. He presented with behavioral changes and short-term memory impairment. He was diagnosed with mild cognitive impairment (MCI) and, later, dementia at the age of 69. He passed away in a nursing home from complications of pneumonia.

\section{Neuropathology}

\section{Methods}

The brains were fixed in formalin and sampled from cortical, subcortical, cerebellar, and brainstem areas according to National Institute on Aging-Alzheimer's Association (NIA-AA), as per the CTE diagnosis consensus paper [17]. Tissue blocks were processed and embedded in paraffin. Six micron sections were stained with Luxol fast blue and hematoxylin and eosin (H\&E/LFB) and by immunohistochemistry with $\gamma \mathrm{H} 2 \mathrm{AX}$ (monoclonal mouse, 1:1000, Ser139, \#05-636;Millipore, MA, USA), phospho-tau (Ser199, Ser202) (polyclonal rabbit, \#44-768G: Thermo Scientific, 1:1000), and TDP-43 (polyclonal rabbit, \#PA5-
29949, Thermo Scientific 1:500), $\beta$-amyloid (monoclonal mouse, DAKO, M0872, 1:50), and $\alpha$-synuclein (monoclonal rabbit, Thermo Scientific \#701085, 1:500).

\section{Gross pathology}

The fresh brain weight of case 1 was normal (1430g) and presented with bilateral cortical contusions in the orbitofrontal areas (plaque jaune) (Fig. 1a, b). This scarring included, bilaterally, the olfactory bulb and tracts (Fig. 1b). On coronal sections there was no evidence of brain atrophy and ventricles were of normal size (Fig. 1c). Case 2 had reduced brain weight $(1243.5 \mathrm{~g})$ and presented with atherosclerosis of the circle of Willis, mild atrophy of the frontal lobes bilaterally, atrophy of the mammillary bodies, dilation of the lateral and third ventricles, fenestrated septum pellucidum and hippocampal atrophy (Fig. 3a-c).

\section{Microscopic pathology}

Case 1 presented with congested microvasculature and patchy, mild perivascular tissue edema with occasional perivascular calcification in the basal ganglia (not shown). Perivascular tau deposits were focal and noted in the parahippocampal gyrus (Fig. 1d (square "a") and E) NFTs were also seen in CA3/4-CA2 (Fig. 1d (square "b") and F). There was no beta-amyloid or TDP-43 aggregates. $\gamma \mathrm{H} 2 \mathrm{AX}$ was widely distributed in neurons and glial cells, in numerous cortical areas (Fig. 2a) with predominance of perivascular distribution (Fig. 2b-d). It was also detected in the brainstem and cerebellum (not shown).

In case 2 , there was pallor of white matter on $H \& E / L F B$ stain (Fig. 3d), severe and widespread p-tau accumulation in neurons and glia without amyloid pathology (Fig. 3e-h, $\mathrm{j}-\mathrm{m})$. Neuronal tau pathology was located primarily in the 
Fig. 2 Distribution of $\gamma \mathrm{H} 2 \mathrm{AX}$ in the frontal cortex of case 1. Panel a shows predominantly perivascular distribution of DNA damage in the cortical (sulcus identified with an arrow in sub-panel a). Asterisks in panel A mark blood vessels to highlight clustering of $\gamma \mathrm{H} 2 \mathrm{AX}$ positive cells near cortical vasculature. Panels b-e show various higher power images of the clustering of $\gamma \mathrm{H} 2 \mathrm{AX}$ in perivascular foci within the frontal cortex
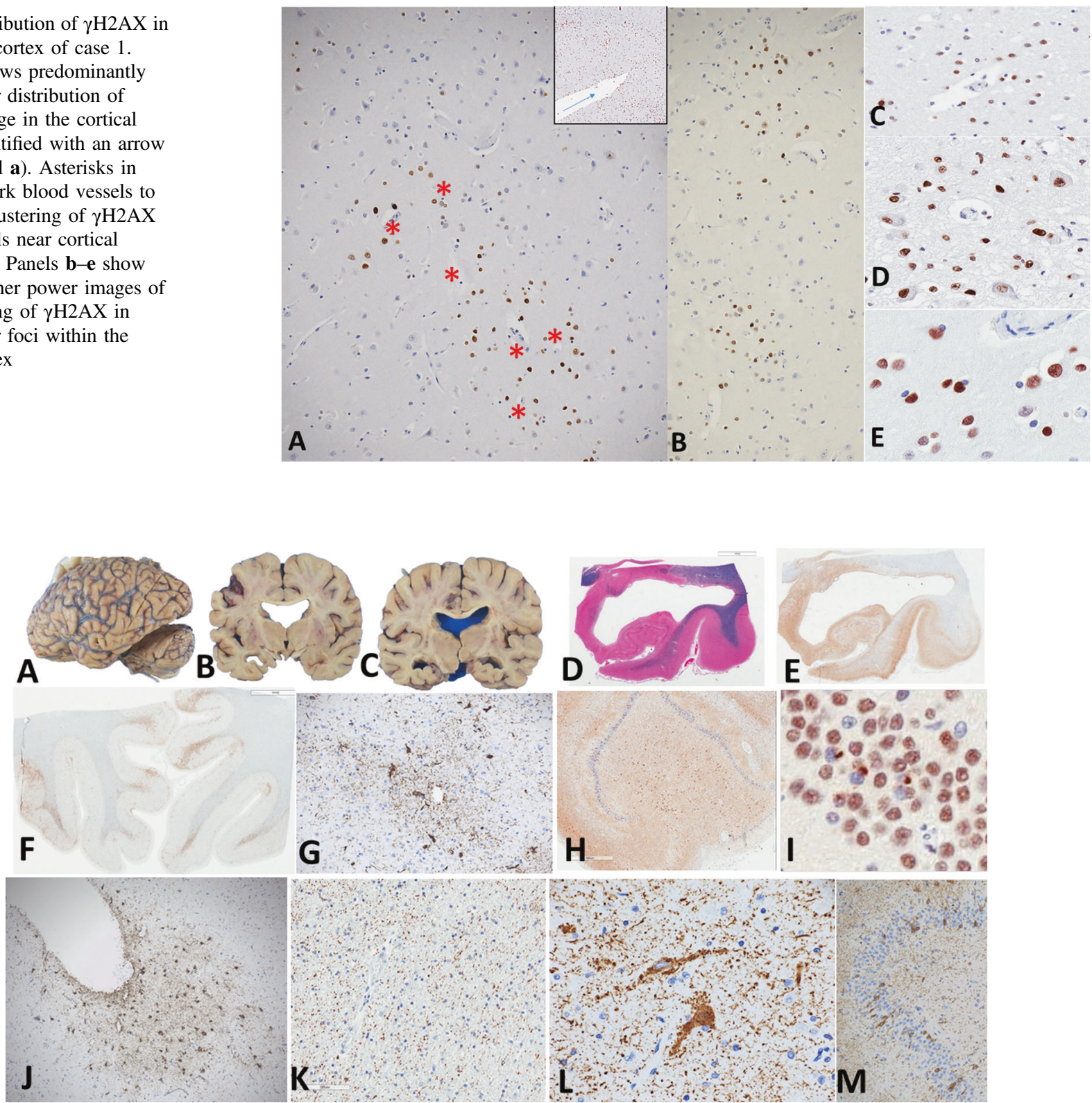

Fig. 3 Gross and microscopic features of case 2. Panels a-c show macroscopic features of the brain with mild cortical atrophy, absence of septum pellucidum and ventricular dilatation (panels b, c). Panels d, e show low power scans of a hippocampal section stained with $\mathrm{H} \& \mathrm{E} /$ luxol fast blue (d) and immuno-labeled with p-tau (e). Panel f shows a low power scan of p-tau distribution in the orbito-frontal cortex. Panel g shows p-tau perivascular deposits in the isocortex. Panel $\mathbf{h}$ shows widespread distribution of $\mathrm{p}$-tau in the hippocampus with high power details in CA3/4 (panel l) and dentate gyrus (panel m). Panel I shows cytoplasmic TDP-43 aggregates in the dentate gyrus. Panel $\mathbf{j}$ shows sub-pial p-tau distribution in the isocortex. Panel $\mathbf{k}$ shows punctate $\mathrm{p}$ tau deposits in the subcortical white matter temporal and frontal cortices, deep gray nuclei, brainstem, and dentate nucleus of the cerebellum. Within the neocortex, neuronal p-tau did not only distribute at the depths of cortical sulci or specific cortical layers, but also at the crown of the gyry (Fig. 3f). Perivascular and sub-pial p-tau deposits were also noted (Fig. 3g, j). Within glial cells, ptau was in the neocortex, temporal structures, deep gray nuclei, and brainstem. The distribution of glial p-tau was in the depths of sulci, subependymal cells, gray matter, white matter, and perivascular regions. There were widespread neuropil threads and diffuse granular staining in the white matter. This individual also had TDP-43 neurites in the superficial cortical layers and neuronal cytoplasmic inclusions in the dentate gyrus (Fig. 3i). $\gamma \mathrm{H} 2 \mathrm{AX}$ was extensive throughout cortical, subcortical, and brainstem regions and was seen in the majority of oligodendrocytes in the white matter, peri-neuronal satellite glial cells, ependymal cells, and sub-ependymal cells (Fig. 4a-d). 
Fig. 4 Immunohistochemistry of $\gamma \mathrm{H} 2 \mathrm{AX}$ in case 2 . Panel a shows a low power view of $\gamma \mathrm{H} 2 \mathrm{AX}$ distribution throughout the hippocampus. Panel b shows a high power view of widespread $\gamma \mathrm{H} 2 \mathrm{AX}$ in oligodendrocytes of the white matter in the hippocampal section shown in panel a. Panel c shows a high power view of DNA damage in the isocortex. $\gamma \mathrm{H} 2 \mathrm{AX}$ is positive in para-neuronal satellite cells (sub-panel C). Panel d shows strong $\gamma \mathrm{H} 2 \mathrm{AX}$ expression in ependymal cells and subventricular glial cells

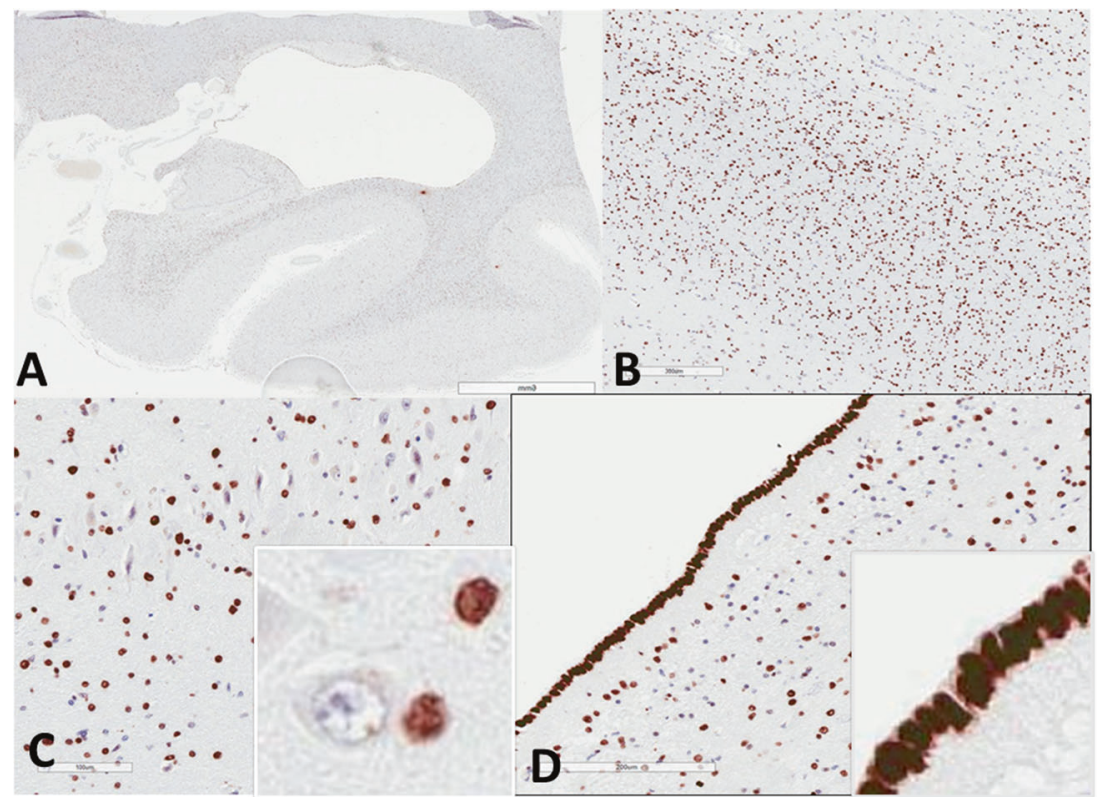

\section{Expression of DDR signaling genes}

\section{Methods}

Our brain samples with a history of mTBI and agedmatched control brains with no mTBI history were selected for gene expression analysis using NanoString Technology, an RNA microarray, well-suited for formalin-fixed tissue and that utilizes molecular "barcodes" to detect and count up to several hundred unique transcripts in one hybridization reaction. Shavings from the hippocampus of each case were used to compare gene expression between cases and controls. Total RNA was isolated from the fixed tissue using the RNeasy FFPE Kit by Qiagen (Qiagen Inc., Toronto, ON, Canada) using the manufacturer's protocol. Total RNA was quantified using the Nanodrop 2000 spectrophotometer (NanoDrop Technologies, Wilmington, DE, USA). Using the digital multiplexed NanoString nCounter analysis system (NanoString Technologies, Seattle, WA, USA), gene expression profiling was then performed on 200 ng RNA from each sample. Raw data was normalized against six housekeeping genes and subsequently analyzed using nSolver solfware (NanoString Technologies). Statistical significance was calculated using normalized RNA count numbers with an unpaired student's $t$-test with statistical significance considered at $p$-values $\leq 0.05$. The mean $\log 2$ fold change was then calculated for each gene.

\section{Increased expression of ATM and CHEK2 in mTBI brains}

The hippocampi of brains with a history of mTBI showed significant upregulation of ATM, the serine/threonine kinase which is activated immediately after DSBs to

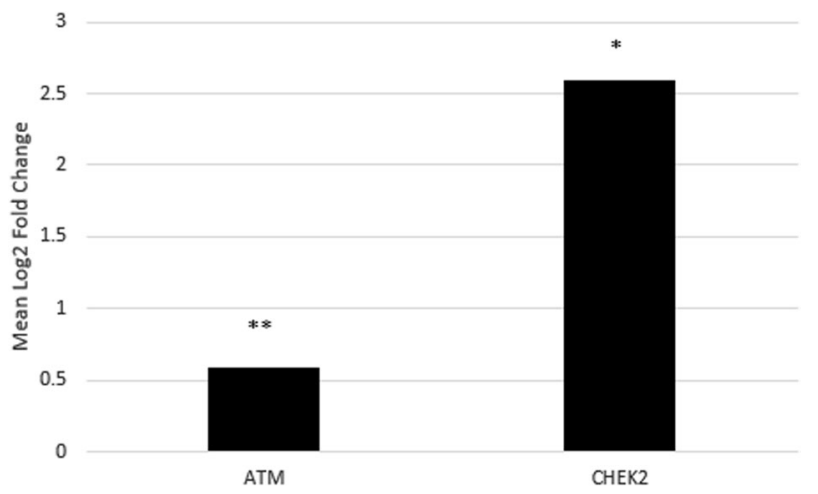

Fig. 5 Mean $\log 2$ fold change of ATM $(p=0.0097)$ and CHEK2 $(p=$ 0.0458 ) expression between hippocampi with a history of mTBI and non-mTBI controls. Statistical significance determined with an unpaired student's $t$-test with significance cut-off at $p \leq 0.05$

orchestrate the DDR and phosphorylate $\mathrm{H} 2 \mathrm{AX}$ into $\gamma \mathrm{H} 2 \mathrm{AX}$. ATM showed an average of a 0.59 -fold increase in expression ( $p=0.0097)$ in mTBI brains compared to healthy controls with no history of mTBI (Fig. 5). CHEK2, a serine/threonine kinase activated by ATM in response to DSBs, was also significantly upregulated in mTBI hippocampi, with an average 2.60-fold increase in expression ( $p$ $=0.0458$ ) across mTBI brains compared to controls (Fig. 5).

\section{Discussion}

The effects of repeated mTBI can be devastating, and some groups such as American football players and boxers can be more affected than others [56, 57]. CTE is therefore an important issue of concern to many athletes, families, and 
clinicians, and a genuine understanding of the underlying molecular driver of concussion pathology will help the development of proper tools for detection of disease in living individuals and targeted effective treatments.

Using $\gamma \mathrm{H} 2 \mathrm{AX}$, we have shown extensive and progressive DNA damage in brains of individuals with recent or remote history of mTBI. We have also provided additional evidence in support of DNA damage. ATM is considered the central component of the DDR, with rapid activation by autophosphorylation immediately after detection of DSBs in the DNA [58]. ATM is therefore a strong marker of the DDR in vivo. This is reflected in the results of our gene expression assay, as both the expression of ATM and its downstream effector CHEK2 were significantly upregulated in mTBI brains. CHEK2 is another serine/threonine kinase, which is activated by ATM in response to DSBs and goes on to activate several proteins involved in the DDR including p53 and BRCA1 [59].

DNA damage can occur in many different forms, including single-nucleotide changes, single-stranded breaks, and double-stranded breaks (DSBs), and a small number of DSBs is sufficient to activate the DDR, a large-scale, dynamic pathway which functions to restore DNA integrity after damage [42]. Accumulation of DNA damage has been associated with neurodegenerative disease and development of neurological symptoms [18] and evidence of DNA damage has been found in brains diagnosed with MCI [60]. This suggests that DNA damage may be a preceding event in the manifestation of AD pathology and other neurodegenerative diseases. In addition, DNA damage has been found in patients with schizophrenia [61], who have interestingly also been reported to have CTE-consistent pathology after surgical axonal injury [62].

Both of the cases presented here had extensive DNA damage throughout various brain regions, including the hippocampus, neocortex, cerebellum, and brainstem. It is important to note that immunoreactivity for $\gamma \mathrm{H} 2 \mathrm{AX}$ extends beyond p-tau pathology in concussed brains. This was especially true in the acute case. Although p-tau burden was comparable to that of $\gamma \mathrm{H} 2 \mathrm{AX}$ in the chronic case, there was a clear increase of $\gamma \mathrm{H} 2 \mathrm{AX}$ load in the second case when compared to the first case. This observation is specifically important, as it indicates that DNA damage may precede abnormal protein aggregation after head trauma, in that $\mathrm{p}$ tau aggregates occur chronically rather than acutely. The pathways leading to neurodegeneration as a result of DNA damage will need further exploration in the future. Another important finding is that that DNA damage expands to more areas with time given the increased load of $\gamma \mathrm{H} 2 \mathrm{AX}$ in the second case, therefore flagging DNA damage as a possible driver of disease progression.

We are therefore proposing markers of DNA damage, for example $\gamma \mathrm{H} 2 \mathrm{AX}$ shown here, as more robust markers of brain damage in individuals with a history of mTBI, as they can capture both the acute and chronic effects of head trauma. DNA damage could represent the first pathological effect of trauma and drive the acute symptoms of mTBI. DNA damage is a reversible condition and can be repaired, however, if individuals have a deficient DNA repair machinery or DNA damage is recurrent and overwhelming the DNA repair pathways, it can become persistent and lead to the proteinopathies noted in chronic mTBI cases.

The mechanism by which DNA damage causes neurodegeneration has not been established here, but it is well known that when the DDR is persistently activated, perhaps in cases of repetitive head trauma or defective DNA repair pathways, cells acquire the senescence-associated secretory phenotype (SASP) [42]. This phenotype is characterized by several hallmark features, including cell-cycle arrest, morphological changes, and secretion of various SASP factors including pro-inflammatory cytokines, proteases, and growth factors [42]. Many of these factors can be found in the blood and CSF, and therefore provides ample opportunity for the discovery of biomarkers relevant to mTBI diagnosis, prognosis, and therapy. These factors have both autocrine and paracrine effects, and this creates a widespread effect far beyond the area of initial damage [63]. The acquisition of a senescent cellular phenotype has been shown to create a chronic state of inflammation often seen in aged brains [64] and, in particular, senescent phenotypes have been reported as playing a role in the progression of neurodegenerative disease [65].

Although DNA damage and SASP have not been characterized in mTBI brains, they have been studied in the context of neurodegenerative disease [66-71]. In a study of $13 \mathrm{AD}$ cases and 8 age-matched controls DNA damage, marked by $\gamma \mathrm{H} 2 \mathrm{AX}$, was found in astrocytic nuclei whereas no DNA damage was found in controls. Interestingly, the damage was not found in neurons with p-tau aggregates, indicating a distinct pathological phenomenon. The authors also pointed out that this damage was consistently localized to the hippocampus, suggesting a clinicopathological correlation to $\mathrm{AD}$ symptoms [72]. Levels of $\gamma \mathrm{H} 2 \mathrm{AX}$ in the blood have also been reported as significantly elevated in $\mathrm{AD}$ patients and $\mathrm{MCI}$ patients compared to healthy controls, suggesting that DNA damage contributes to the pathology of $\mathrm{AD}$ and early cognitive symptoms [73]. In another study focused on $\mathrm{AD}$, robust markers of the SASP, including $\mathrm{p} 16^{\mathrm{INK} 4 \mathrm{a}}$ and matrix metalloproteinase-1 (MMP-1), were significantly increased in astrocytes in the frontal cortices of AD patients compared to controls. DNA damage, marked by $\gamma \mathrm{H} 2 \mathrm{AX}$ and DNA-dependent protein kinase catalytic subunit (DNA-PKcs), and senescence, marked by $\beta$-galactosidase, are increased in AD brains compared to controls, suggesting a role of DNA damage and succeeding senescence in the progression of AD [74]. From the abundance of 
evidence reporting DNA damage and senescence in AD it is therefore conceivable that the same molecular mechanisms underlie mTBI-induced symptoms and neurodegeneration.

Overall we propose that DNA damage alone, and possibly subsequent acquisition of SASP (not studied here), cause functional changes that may be the underlying basis of symptoms seen after mTBI in both acute and chronic cases. This pathological change results in altered gene expression which affects many aspects of brain health, contributing to early-onset brain aging and neurodegeneration. We therefore suggest that in brains with a history of mTBI, DNA damage precedes proteinopathy and induces a senescent phenotype, which may cause by itself the broadspectrum symptoms and neuropathology in these individuals.

It is important to note that the cause of pathological changes including DNA damage is not clear. We postulate that any insult causing oxidative stress can induce the DNA damage. Therefore, DNA damage can be a common but important pathway in multiple scenarios such substance abuse or chronic systemic disease. Indeed, administration of cocaine into rats has been shown to induce DNA damage and tau hyperphosphorylation in the hippocampus, cortex, and caudatoputamen [75]. It has also been reported, in humans, that heavy drinking is associated with faster cognitive decline in $\mathrm{AD}$ [76]. As is similar with the possibly misconstrued correlation between p-tau pathology and mTBI symptoms, we must therefore be cautious with the readiness with which we establish cause and significance of pathology. Animal studies will be more suited to directly establish causality in all of these situations.

\section{Conclusion}

Here we propose that markers of DNA damage are robust indicators of brain damage in athletes with a history of repetitive mTBIs compared to p-tau. Two cases of acute and chronic mTBI presented in this paper have shown DNA damage accumulation which probably preceded p-tau pathology and other protein aggregations, as has been reported previously in the literature [77]. The cause of pathology in these individuals is not entirely clear, as they had history of chronic systemic disease or history of drug and alcohol use. This paper suggests that the p-tau seen and used as a diagnostic marker in CTE is most likely the endstage pathology of a neurodegenerative disease caused by head trauma, whereas DNA damage is the mechanism by which these pathological entities emerge. Persistent DNA damage, in the case of repetitive head trauma, may be sufficient for acquiring SASP, a chronic state of inflammation characteristic of aged and neurodegenerative brains. These early-stage phenomena represent additional robust markers of brain damage in athletes as they may provide a sufficient explanation of clinical symptoms, point out pathophysiological mechanisms of p-tau accumulation, explain the high incidence of co-morbid neurodegenerative diseases with CTE, and ultimately provide potential therapeutic, diagnostic, and prognostic biomarkers for mTBIinduced brain damage.

Acknowledgements We would like to thank Road2Recovery and the Canadian Concussion Centre for funding of this research.

\section{Compliance with ethical standards}

Conflict of interest The authors declare that they have no conflict of interest.

Publisher's note: Springer Nature remains neutral with regard to jurisdictional claims in published maps and institutional affiliations.

\section{References}

1. Carroll LJ, Cassidy JD, Peloso PM, et al. Prognosis for mild traumatic brain injury: results of the WHO collaborating centre task force on mild traumatic brain injury. J Rehabil Med. 2004;43 (Suppl):84-105.

2. Gardner RC, Yaffe K. Epidemiology of mild traumatic brain injury and neurodegenerative disease. Mol Cell Neurosci. 2015;66 (Pt B):75-80.

3. Coburn K. Traumatic brain injury: the silent epidemic. AACN Clin Issues Crit Care Nurs. 1992;3:9-18.

4. Bergman K, Given B, Fabiano R, et al. Symptoms associated with mild traumatic brain injury/concussion: the role of bother. $\mathrm{J}$ Neurosci Nurs. 2013;45:124-32.

5. Stern RA, Daneshvar DH, Baugh CM, et al. Clinical presentation of chronic traumatic encephalopathy. Neurology. 2013;81: 1122-9.

6. Marshall S, Bayley M, McCullagh S, et al. Updated clinical practice guidelines for concussion/mTBI and persistent symptoms. Brain Inj. 2015;29:688-700.

7. Towns SJ, Silva MA, Belanger HG. Subjective sleep quality and postconcussion symptoms following mTBI. Brain Inj. 2015;29:1337-41.

8. Mortimer JA, van Duijn CM, Chandra V, et al. Head trauma as a risk factor for Alzheimer's disease: a collaborative re-analysis of case-control studies. Int J Epidemiol. 1991;20(Suppl 2): S28-35.

9. Jafari S, Etminan M, Aminzadeh F, et al. Head injury and risk of Parkinson's disease: a systematic review and meta-analysis. Mov Disord. 2013;28:1222-9.

10. Kalkonde YV, Jawaid A, Qureshi SU, et al. Medical and environmental risk factors associated with frontotemporal dementia: a case-control study in a veteran population. Alzheimer's Dement. 2012;8:204-10.

11. Chen H, Richard M, Sandler DP, et al. Head injury and amyotrophic lateral sclerosis. Am J Epidemiol. 2007;166:810-6.

12. Adams JW, Alvarez VE, Mez J, et al. Lewy body pathology and chronic traumatic encephalopathy associated with contact sports. J Neuropathol Exp Neurol. 2018;77:757-68.

13. Nordstrom A, Nordstrom P. Traumatic brain injury and the risk of dementia diagnosis: a nationwide cohort study. PLoS Med. 2018;15:e1002496. 
14. Barnes DE, Byers AL, Gardner RC, et al. Association of mTBI with and without LOC with dementia in US military veterans. JAMA Neurol. 2018;75:1055-61.

15. McKee AC, Stern RA, Nowinski CJ, et al. The spectrum of disease in chronic traumatic encephalopathy. Brain. 2013;136(Pt.1):43-64.

16. Mez J, Daneshvar DH, Kiernan PT, et al. Clinicopathological evaluation of chronic traumatic encephalopathy in players of American football. JAMA. 2017;318:360-70.

17. McKee AC, Cairns NJ, Dickson DW, et al. The first NINDS/ NIBIB consensus meeting to define neuropathological criteria for the diagnosis of chronic traumatic encephalopathy. Acta Neuropathol. 2016;131:75-86.

18. Maynard S, Fang EF, Scheibye-Knudson M, et al. DNA damage, DNA repair, aging, and neurodegeneration. Cold Spring Harb Perspect Med. 2015;5:pii a025130.

19. Stein TD, Montenigro PH, Alvarez VE, et al. Beta amyloid deposition in chronic traumatic encephalopathy. Acta Neuropathol. 2015;130:21-34.

20. Walsh DM, Teplow DB. Alzheimer's disease and the amyloid $\beta$ protein. Prog Mol Biol Transl Sci. 2012;107:101-24.

21. Uryu K, Chen XH, Martinez D, et al. Multiple proteins implicated in neurodegenerative diseases accumulate in axons after brain trauma in humans. Exp Neruol. 2007;208:185-92.

22. Villar Pique A, Lopes da Fonseca T, Outeiro TF. Structure, function, and toxicity of alpha-synuclein: the Bermuda triangle in synucleinopathies. J Neurochem. 2016;139(Suppl 1):240-55.

23. Uryu K, Giasson BI, Longhi L, et al. Age-dependent synuclein pathology following traumatic brain injury in mice. Exp Neurol. 2003;184:214-24.

24. Mondello S, Buki A, Italiano D, et al. a-synuclein in CSF of patients with severe traumatic brain injury. Neurology. 2013;80:1662-8.

25. Gomperts SN. Lewy body dementias: dementia with lewy bodies and parkinson disease dementia. Continuum. 2016;22(2 Dementia):435-63.

26. Tan RH, Kril JJ, Fatima M, et al. TDP-43 proteinopathies: pathological identification of brain regions differentiating clinical phenotypes. Brain. 2015;138(Pt 10):3110-22.

27. Sweeney P, Park H, Baumann M, et al. Protein misfolding in neurodegenerative diseases: implications and strategies. Transl Neurodegener. 2017;13:6.

28. Stefanis L. a-synuclein in Parkinson's disease. Cold Spring Harb Perspect Med. 2012;2:a009399.

29. Neumann M, Sampathu DM, Kwong LK, et al. Ubiquitinated TDP-43 in frontotemporal lobar degeneration and amyotrophic lateral sclerosis. Science. 2006;314:130-3.

30. McKee AC, Daneshvar DH. The neuropathology of traumatic brain injury. Handb Clin Neurol. 2015;127:45-66.

31. Washington PM, Villapol S, Burns MP. Polypathology and dementia after brain trauma: does brain injury trigger distinct neurodegenerative diseases, or should they be classified together as traumatic encephalopathy? Exp Neurol. 2016;275:381-8.

32. Roman P, Ruiz-Cantero A. Polypathology and emerging phenomenon and a challenge for healthcare systems. Rev Clin Esp. 2017;217:229-37.

33. Gao A, Ramsay D, Twose R, et al. Chronic traumatic encephalopathy-like neuropathological findings without a history of trauma. Int J Pathol Clin Res. 2017;3:050.

34. Kovacs GG, Ferrer I, Grinberg LT, et al. Aging-related tau astrogliopathy (ARTAG): harmonized evaluation strategy. Acta Neuropathol. 2016;131:87-102.

35. Asken BM, Sullan MJ, DeKosky ST, et al. Research gaps and controversies in chronic traumatic encephalopathy: a review. JAMA Neurol. 2017;74:1255-62.

36. Schwab N, Hazrati LN. Current understanding of chronic traumatic encephalopathy. J Alzheimers Dis. 2018;64:1067-76.
37. McKinnon PJ. DNA repair deficiency and neurological disease. Nat Rev Neurosci. 2009;10:100-12.

38. Fishel ML, Vasko MR, Kelley MR. DNA repair in neurons: so if they don't divide what's to repair? Mutat Res. 2007;614:24-36.

39. Mullaart E, Boerrigter ME, Ravid R, et al. Increased levels of DNA breaks in cerebral cortex of AD patients. Neurobiol Aging. 1990;11:169-73.

40. Coppede F, Migliore L. DNA damage and repair in Alzheimer's disease. Curr Alzheimer Res. 2009;6:36-47.

41. Wang J, Markesbery WR, Lovell MA. Increased oxidative damage in nuclear and mitochondrial DNA in mild cognitive impairment. J Neurochem. 2006;96:825-32.

42. Fielder E, von Zglinicki T, Jurk D. The DNA damage response in neurons: die by apoptosis or survive in a senescence-like state? J Alzheimers Dis. 2017;60(s1):S107-31.

43. Jackson SP, Bartek J. The DNA damage response in human biology and disease. Nature. 2009;461:1071-8.

44. Wang Y, Arun P, Wei Y, et al. Repeated blast exposures cause brain fragmentation in mice. J Neurotrauma. 2014;31:498-504.

45. Ohayon S, Boyko M, Saad A, et al. Cell-free DNA as a marker for prediction of brain damage in traumatic brain injury in rats. J Neurotrauma. 2012;29:261-7.

46. Rogaku E, Nieves-Neira W, Boon C, et al. Initiation of DNA fragmentation during apoptosis induces phosphorylation of $\mathrm{H} 2 \mathrm{AX}$ histone at serine 139. J Biol Chem. 2000;275:9390-5.

47. Ishiai M, Uchida E, Takata M. Establishment of the DNA repairdefective mutants in DT40 cells. Methods Mol Biol. 2012;920:39-49.

48. Huang X, Traganos F, Darzynkiewicz Z. DNA damage induced by DNA topoisomerase I- and topoisomerase II-inhibitors detected by histone $\mathrm{H} 2 \mathrm{AX}$ phosphorylation in relation to the cell cycle phase and apoptosis. Cell Cycle. 2003;2:614-9.

49. Turinetto V, Giachino C. Multiple facets of histone variant $\mathrm{H} 2 \mathrm{AX}$ : a DNA double-strand-break marker with several biological functions. Nucleic Acids Res. 2015;43:2489-98.

50. Pilch DR, Sedelnikova OA, Redon C, et al. Characteristics of gamma-H2AX foci at DNA double-strand break sites. Biochem Cell Biol. 2003;81:123-9.

51. Kinner $\mathrm{A}, \mathrm{Wu} \mathrm{W}$, Staudt $\mathrm{C}$, et al. Gamma-H2AX in recognition and signaling of DNA double-strand breaks in the context of chromatin. Nucleic Acids Res. 2008;36:5678-94.

52. Mei L, Hu Q, Peng J, et al. Phospho-histone H2AX is a diagnostic and prognostic marker for epithelial ovarian cancer. Int J Clin Exp Pathol. 2015;8:5597-802.

53. Marachal A, Zou L. DNA damage sensing by the ATM and ATR kinases. Cold Spring Harb Perspect Biol. 2013;5:a012716.

54. Kitagawa R, Kastan MB. The ATM-dependent DNA damage signaling pathway. Cold Spring Harb Symp Quant Biol. 2005;70:99-109.

55. Bakkenist CJ, Kastan MB. DNA damage activates ATM through intermolecular autophosphorylation and dimer dissociation. Nature. 2003;421:499-506.

56. CTE found in $99 \%$ of studied brains from deceased NFL players. CNN, 2017. https://www.cnn.com/2017/07/25/health/cte-nfl-pla yers-brains-study/index.html

57. Another young NFL player calls it quits to protect future health. The Seattle Times, 2015. https://www.seattletimes.com/sports/seahaw ks/another-young-nfl-player-calls-it-quits-to-protect-future-health/

58. Burma S, Chen BP, Murphy M, et al. ATM phosphorylates histone $\mathrm{H} 2 \mathrm{AX}$ in response to DNA double-strand breaks. J Biol Chem. 2001;276:42462-7.

59. Zannini L, Deli D, Buscemi G. CHK2 kinase in the DNA damage response and beyond. J Mol Cell Biol. 2014;6:442-57.

60. Lovell MA, Markesbery WR. Oxidative DNA damage in mild cognitive impairment and late-stage Alzheimer's disease. Nucleic Acids Res. 2007;35:7497-504. 
61. Nishioka N, Arnold SE. Evidence for oxidative DNA damage in the hippocampus of elderly patients with chronic schizophrenia. Am J Geriatr Psychiatry. 2004;12:167-75.

62. Shively SB, Edgerton SL, Iacono D, et al. Localized cortical chronic traumatic encephalopathy pathology after single, severe axonal injury in human brain. Acta Neuropathol. 2017;133:353-66.

63. Coppe JP, Desprez PY, Krtolica A, et al. The senescenceassociated secretory phenotype: the dark side of tumor suppression. Annu Rev Pathol. 5:99-118.

64. Franceschi C, Capri M, Monti D, et al. Inflammaging and antiinflammaging: a systematic perspective on aging and longevity emerged from studies in humans. Mech Ageing Dev. 2007;128: 92-105.

65. Bhat R, Crowe EP, Bitto A, et al. Astrocyte senescence as a component of Alzheimer's disease. PLoS ONE. 2012;7:e45069.

66. Simpson JE, Ince PG, Haynes LJ, et al. Population variation in oxidative stress and astrocyte DNA damage in relation to Alzheimer-type pathology in the ageing brain. Neuropathol Appl Neurobiol. 2010;36:25-40.

67. Simpson JE, Ince PG, Matthews FE, et al. Neuronal DNA damage response-associated dysregulation of signaling pathways and cholesterol metabolism at the earliest stages of Alzheimer-type pathology. Neuropathol Appl Neurobiol. 2016;42:167-79.

68. Francois M, Leifert WR, Hecker J, et al. Guanine-quadruplexes are increased in mild cognitive impairment and correlate with cognitive function and chromosomal DNA damage. DNA Repair. 2016;46:29-36.

69. Sepe S, Milanese C, Gabriels S, et al. Inefficient DNA repair is an aging-related modifier of Parkinson's Disease. Cell Rep. 2016;15:1866-75.
70. Raj D, Jaarsma D, Holtman IR, et al. Priming of microglia in a DNA-repair deficient model of accelerated aging. Neurobiol Aging. 2014;35:2147-60.

71. Yu HarrisonFE, Xia F. Altered DNA repair; an early pathogenic pathway in Alzheimer's disease and obesity. Sci Rep. 2018; 8:5600.

72. Myung NH, Zhu X, Kruman II, et al. Evidence of DNA damage in Alzheimer disease: phosphorylation of histone H2AX in astrocytes. Age (Dordr). 2008;30:109-15.

73. Siddiqui MS, Francois M, Hecker J, et al. gamma-H2AX is increased in peripheral blood lymphocytes of Alzheimer's disease patients in the South Australian neurodegeneration, nutrition, and DNA damage (SAND) study of aging. Mutat Res. 2018; 829-30:6-18.

74. Garwood CJ, Simpson JE, Al Mashhadi S, et al. DNA damage response and senescence in endothelial cells of human cerebral cortex and relation to Alzheimer's neuropathology progression: a population based study in the medican research council cognitive function and ageing study (MRC-CFAS) cohort. Neuropathol Appl Neurobiol. 2014;40:802-14.

75. Liu SJ, Fang ZY, Yang Y, et al. Alzheimer-like phophorylation of tau and neurofilament induced by cocaine in vivo. Acta Pharmacol Sin. 2003;24:512-8.

76. Heymann D, Stern Y, Costentino S, et al. The association between alcohol use and the progression of Alzheimer's disease. Curr Alzheimer Res. 2016;13:1356-62.

77. Su JH, Deng G, Cotman CW. Neuronal DNA damage precedes tangle formation and is associated with up-regulation of nitrotyrosine in Alzheimer's disease brain. Brain Res. 1997;774: 193-9. 